\title{
Superlattice of Single Atom Magnets on Graphene
}

\author{
Romana Baltic, ${ }^{\dagger}$ Marina Pivetta, ${ }^{\dagger}$ Fabio Donati, ${ }^{\dagger}$ Christian Wäckerlin, ${ }^{\dagger}$ Aparajita Singha, ${ }^{\dagger}$ Jan Dreiser, ${ }^{\dagger, \ddagger}$ \\ Stefano Rusponi, ${ }^{\dagger}$ and Harald Brune ${ }^{* \dagger}$ \\ ${ }^{\dagger}$ Institute of Physics, Ecole Polytechnique Fédérale de Lausanne, Station 3, CH-1015 Lausanne, Switzerland \\ ${ }^{\ddagger}$ Swiss Light Source, Paul Scherrer Institute, CH-5232 Villigen PSI, Switzerland
}

\section{Supporting Information}

ABSTRACT: Regular arrays of single atoms with stable magnetization represent the ultimate limit of ultrahigh density storage media. Here we report a self-assembled superlattice of individual and noninteracting Dy atoms on graphene grown on $\operatorname{Ir}(111)$, with magnetic hysteresis up to $5.6 \mathrm{~T}$ and spin lifetime of $1000 \mathrm{~s}$ at $2.5 \mathrm{~K}$. The observed magnetic stability is a consequence of the intrinsic low electron and phonon densities of graphene and the 6-fold symmetry of the adsorption site. Our array of single atom magnets has a density of $115 \mathrm{Tbit} / \mathrm{inch}^{2}$, defined by the periodicity of the graphene moire pattern.

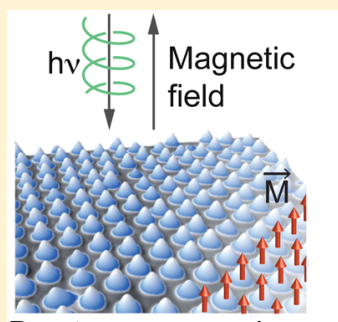

Dy atoms on graphene

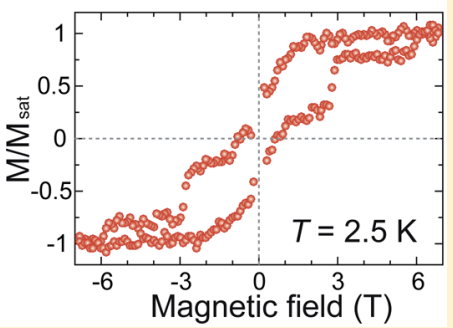

KEYWORDS: Single atom magnets, self-assembly, superlattice, rare earth atoms, graphene, XMCD

$\mathrm{T}$ he fabrication of ordered structures at the nanoscale is a crucial step toward information storage at ultimate length scales. ${ }^{1,2}$ Realizing highly ordered and monodispersed magnetic structures stands as one of the key challenges for increasing the bit density of magnetic storage devices. The ultimate limit of a single atom per bit guarantees the highest storage density and minimal dipolar coupling among the bits. Single-ion molecular magnets, ${ }^{3}$ as well as metal-organic networks, ${ }^{4}$ allow the selfassembly of single magnetic atoms in ultradense arrays. The molecular cage defines the spacing between the magnetic cores and can protect them from contamination. However, the coupling with electrons and vibrational modes of the surrounding ligands limits the magnetic stability of the magnetic core presently to temperatures below $20 \mathrm{~K}$ in bulk ${ }^{5}$ and $8 \mathrm{~K}$ for surface supported molecules. ${ }^{6}$ The absence of the organic ligand, i.e., having individual atoms adsorbed on the surface, may result in a reduced interaction with the environment and greater magnetic stability. Intense research on the magnetism of single atoms ${ }^{7-12}$ has culminated in the achievement of magnetic remanence in Ho atoms randomly adsorbed onto $\mathrm{MgO}$, with magnetic stability up to $40 \mathrm{~K}^{13}$ Reading and writing of these atoms has recently been demonstrated. ${ }^{14}$ Nevertheless, so far the realization of an ensemble of single atoms combining long magnetic lifetimes with spatial order has remained elusive and stands as the next milestone. Here we exploit the selective adsorption of Dy atoms in the periodic moiré pattern formed by graphene on lattice mismatched $\operatorname{Ir}(111)^{15}$ to create a superlattice of single atom magnets with a mean distance of $2.5 \mathrm{~nm}$ and negligible mutual magnetic interactions.

Ensembles of individual Dy atoms on graphene on $\operatorname{Ir}(111)$ are obtained by deposition with an $e$-beam evaporator (Figure 1a, middle). The spatial arrangement of these atoms on the graphene moire pattern can be controlled by the sample temperature during deposition, $T_{\text {dep }}$. Deposition below $10 \mathrm{~K}$ yields statistical growth with a random distribution of Dy atoms, as demonstrated by scanning tunneling microscopy (STM) in Figure 1a, left. At $40 \mathrm{~K}$, surface diffusion of Dy is activated and, therefore, the atoms can reach the most favorable adsorption site in the moire unit cell, namely, where the C-ring is situated atop an Ir atom. This yields a well-ordered Dy superlattice with one atom every $(10 \times 10)$ graphene unit cells (Figure 1a, right). The mechanism giving rise to this remarkable long-range order is further detailed in the Supporting Information, where we also show a large scale STM image and its autocorrelation function.

The magnetic properties of Dy atoms in both spatial arrangements were investigated with X-ray absorption spectroscopy (XAS) and magnetic circular dichroism (XMCD). Spectra obtained at the $M_{4,5}$ edges $(3 \mathrm{~d} \rightarrow 4 \mathrm{f}$ transitions $)$ and at $T=2.5 \mathrm{~K}$ with collinear X-ray beam and external magnetic field $B$ are shown in Figure 2 for two different incidence angles $\theta$. The maximum of the XMCD intensity is proportional to the projection of the total magnetic moment along the beam axis. Hence, by recording this maximum (Figure $2 \mathrm{a}$ ) as a function of the external magnetic field, one can acquire a magnetization curve of the system. The out-of-plane magnetization curves $(\theta$ $\left.=0^{\circ}\right)$ in Figure $1 \mathrm{~b}$ show clear hysteresis extending up to $B=$ $\pm 5.6 \mathrm{~T}$. Since we measure in total electron yield (TEY) mode, the data points are highly scattered for small fields $(-0.2 \mathrm{~T} \leq B$ $\leq 0.2 \mathrm{~T})$. Assuming that $M(B)$ is linear in that region, we estimate a magnetic remanence of approximately $30 \%$ of the

Received: August 23, 2016

Revised: October 21, 2016

Published: October 25, 2016 

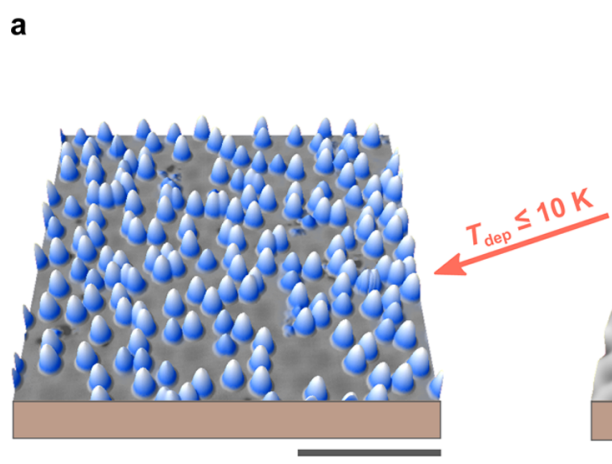

b

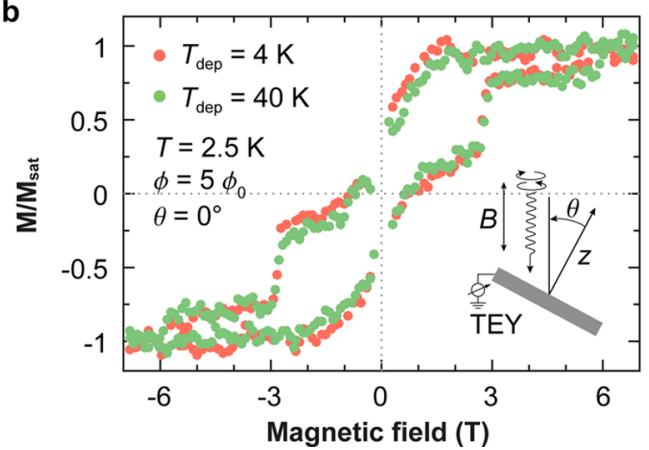

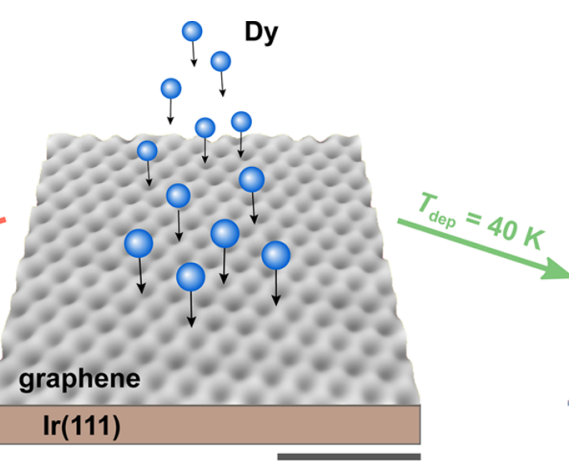

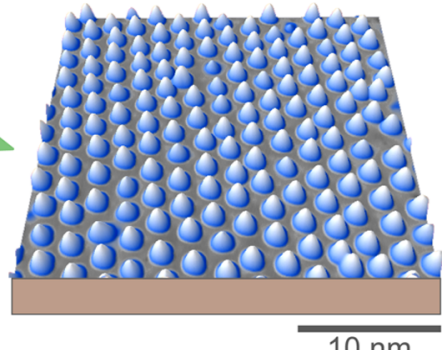

$10 \mathrm{~nm}$

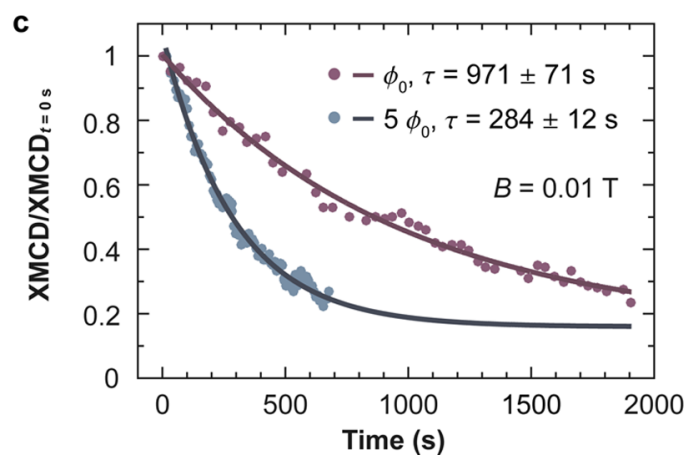

Figure 1. Growth and magnetism of a superlattice of Dy atoms on graphene/Ir(111). (a) Middle, STM image of graphene/Ir(111) moiré with schematic deposition of Dy; left, randomly distributed Dy atoms upon deposition at $10 \mathrm{~K}$; right, well-ordered superlattice of Dy atoms upon deposition at $40 \mathrm{~K}$; the Dy atoms ( $83 \%$ of the objects on the surface for both $\left.T_{\text {dep }}\right)$ have an apparent height of $0.50 \pm 0.02 \mathrm{~nm}\left(\right.$ left, sample bias $V_{\mathrm{t}}=$ $-0.5 \mathrm{~V}$, tunnel current $I_{\mathrm{t}}=20 \mathrm{pA}$; middle, $V_{\mathrm{t}}=-0.05 \mathrm{~V}, I_{\mathrm{t}}=50 \mathrm{pA}$; right, $V_{\mathrm{t}}=-0.2 \mathrm{~V}, I_{\mathrm{t}}=100 \mathrm{pA} ; T=5 \mathrm{~K}$, Dy coverage $\Theta_{\mathrm{Dy}}=0.01 \mathrm{ML}$, with one monolayer (ML) being one Dy atom per graphene unit cell). (b) Magnetization curves of the Dy superlattice (green) and randomly distributed Dy atoms (red) $\left(T=2.5 \mathrm{~K}\right.$, incidence angle $\theta=0^{\circ}$, photon flux $\phi=5 \phi_{0}$, with $\phi_{0}=3 \times 10^{-3}$ photons $\left.\mathrm{nm}^{-2} \mathrm{~s}^{-1}, \dot{B}=33 \mathrm{mT} \mathrm{s}{ }^{-1}\right)$. Inset: field, beam, and sample geometry. (c) Time evolution of the maximum XMCD intensity at $0.01 \mathrm{~T}$ acquired with $\phi_{0}$ (purple dots) and $5 \phi_{0}$ (blue dots) after saturation of the sample magnetization at $6.8 \mathrm{~T}$. Exponential fits (solid lines) give the magnetic relaxation time $\tau$.

saturation magnetization $M_{\text {sat }}$. These hysteresis loops also show several steps that are characteristic of quantum tunneling of the magnetization (QTM) due to crossing of quantum levels at the respective external magnetic fields. ${ }^{16}$ These steps will be discussed later in more detail. The magnetization curves acquired on the superlattice and on the randomly distributed atoms are identical within our resolution. This demonstrates, first, that the magnetic properties of Dy atoms are not influenced by their position within the moiré pattern. Second, since the two systems have different Dy-Dy distance distributions, mutual interactions, either dipolar or substratemediated Ruderman-Kittel-Kasuya-Yosida (RKKY), are not significant and in particular not responsible for the observed slow magnetic relaxation.

The magnetic lifetime of the Dy atoms is investigated by acquiring the decay time of the maximum XMCD intensity after saturating the sample magnetization in an external field of $B=6.8 \mathrm{~T}$ and subsequently reducing it close to zero $(B=0.01$ $\mathrm{T})$. At the $\mathrm{X}$-ray flux $\phi$ used to acquire the magnetization curves, $\phi=5 \phi_{0}$, the lifetime is $284 \pm 12 \mathrm{~s}$ (Figure 1c). However, reducing the X-ray flux by a factor of 5 increases the lifetime to $971 \pm 71 \mathrm{~s}$. Similar flux dependence of the magnetic lifetimes was observed for $\mathrm{Ho} / \mathrm{MgO}^{13}$ and for molecular magnets, ${ }^{6,17}$ and was ascribed to X-ray induced secondary electrons destabilizing the magnetic quantum states. Therefore, the larger of the two values above represents only the lower bound for the intrinsic magnetic lifetime of Dy atoms.

The observation of long magnetic lifetimes indicates that the Dy quantum states are well protected against QTM and direct scattering with electrons and phonons of the surface. In order to identify the symmetry and the splitting of the magnetic states, we compared the experimental XAS, XMCD, and magnetic linear dichroism (XMLD) spectra with multiplet calculations performed using the multiX code ${ }^{18}$ (see Figure $2 b$ and Supporting Information). These calculations include the effect of external magnetic field, finite temperature, incidence angle of X-rays, and crystal field ( $\mathrm{CF}$ ) environment of the magnetic atom. As identified by atomic resolution STM images (see Supporting Information), the Dy atoms adsorb in the 6fold symmetrical $\left(C_{6 v}\right)$ hollow sites of the hexagonal carbon rings. Therefore, we simulated the effect of the graphene $\mathrm{CF}$ with a $C_{6 v}$ arrangement of point charges around the Dy atom (see Figure S6a).

The calculations reveal $4 \mathrm{f}^{10}$ occupation and $J=8$ total angular momentum, as for Dy atoms in the gas phase. The adsorption on graphene promotes strong uniaxial anisotropy and a magnetic ground state with out-of-plane projected total moment $J_{z}= \pm 7$ (Figure 2c). In the $C_{6 v}$ symmetry of the CF, magnetic quantum states differing by $\Delta J_{z}= \pm 6$ are mixed, hence forming six classes of eigenstates depicted with six different colors in Figure 2c. In particular, the CF strongly mixes states with $J_{z}= \pm 6$ and $J_{z}= \pm 3$, resulting in tunnel-split doublets of almost quenched $J_{z}$. The $J_{z}= \pm 6$ states reduce the effective magnetic anisotropy barrier at $B=0 \mathrm{~T}$ from $21.4 \mathrm{meV}$ to only $5.6 \mathrm{meV}$, thus offering a thermally activated shortcut for the magnetic relaxation of the Dy atoms.

The remarkable magnetic stability stems from the combination of the ground state and adsorption site symmetry. At $B=0$ 

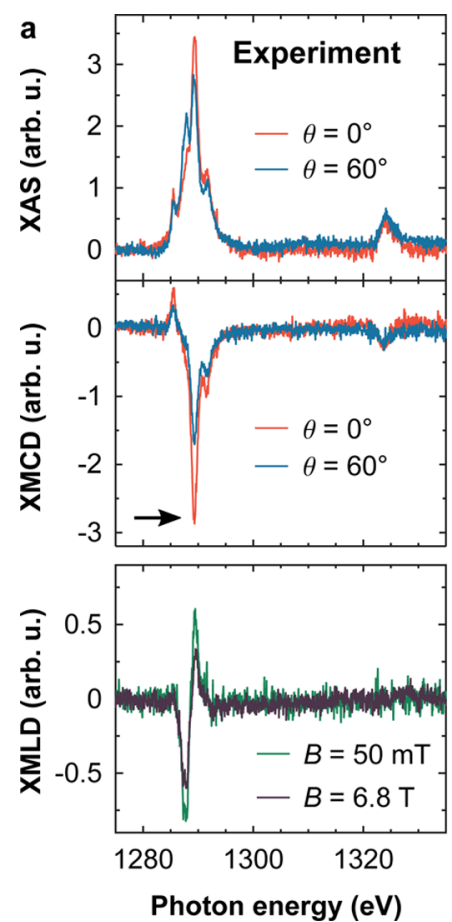

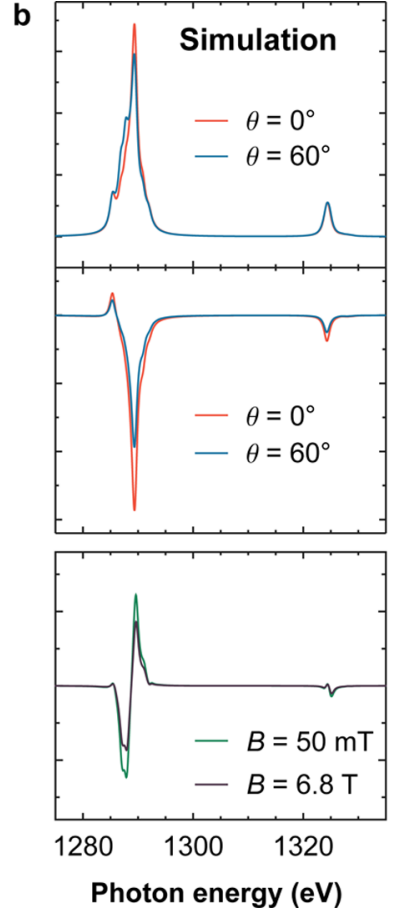

Figure 2. Absorption spectra, magnetic ground state, and zero-field splitting of Dy/graphene/Ir(111). (a) XAS, XMCD, and XMLD spectra measured at the $M_{4,5}$ Dy edges. The black arrow points to the peak at $1289.3 \mathrm{eV}$ where the magnetization curve and relaxation time are measured (B $=6.8 \mathrm{~T}, T=2.5 \mathrm{~K}$ ). (b) XAS, XMCD, and XMLD spectra resulting from multiplet calculations for single Dy atoms in the $C_{6 v}$ CF of graphene. (c) Zero-field splitting of the Dy lowest multiplet $(J=8)$ resulting from multiplet calculations. The splitting at $J_{z}=0$ is emphasized for better clarity. Each color identifies magnetic quantum states belonging to one of the six classes defined by the $C_{6 v}$ group symmetry; dashed lines connect doublets with quenched $J_{z}$.

$\mathrm{T}$, the CF does not mix $J_{z}= \pm 7$ states, hence the doublet is degenerate and no QTM occurs. In addition, in the $C_{6 v}$ symmetry first-order scattering $\left(\Delta J_{z}= \pm 1\right)$ with conduction electrons and phonons cannot induce direct transition between states with $J_{z}=7$ and $J_{z}=-7 .{ }^{19,20}$ Even considering coupling with nuclear spins, i.e., hyperfine interactions, the Dy atoms are protected from QTM for the following reasons: first, more than half of the isotopes of Dy ( $56.2 \%$ natural abundance) have no nuclear spin $(I=0)$. Second, all the other isotopes have a nuclear spin $I=5 / 2$ and its coupling to the integer total electron moment leads to a half integer spin, for which QTM is forbidden due to Kramers' theorem. Thus, the magnetization can only reverse via thermal excitations to the first tunnel-split doublet with $J_{z}= \pm 6$ at $B=0 \mathrm{~T}$ (Figure 2c), and to the top of the energy barrier in finite fields. This explains the opening of the magnetization curve up to large fields. Additional relaxation can occur due to QTM induced by the hyperfine coupling for finite fields below $20 \mathrm{mT}^{21}$

The sharp jumps in the magnetization curve can be rationalized by the field-dependent energy splitting of the Dy quantum levels. Figure $3 \mathrm{~b}$ shows the Zeeman diagram of the lowest lying Dy states for the range of magnetic fields used in our experiments. Several level crossings occur, but at $2.5 \mathrm{~K}$ only the lowest levels are thermally populated and only their crossings have an effect on the magnetization curve. As discussed above, at $B=0 \mathrm{~T}$, the direct transition between the two ground states $J_{z}= \pm 7$ is forbidden and occurs via thermal excitation to the $J_{z}= \pm 6$ states. At $B=2.7 \mathrm{~T}$, levels $J_{z}=7$ and $J_{z}$ $=-8$ cross; however, QTM should not occur since the two states do not mix in the $C_{6 v}$ symmetry of the CF. The only way to rationalize the sharp step observed in the magnetization curve is to assume a small $C_{3 v}$ term in the $\mathrm{CF}$, not included in the calculations and arising, for example, from the nonequivalence of the $\mathrm{A}-\mathrm{B}$ carbon sublattices, which affects the electron scattering processes in graphene. ${ }^{22,23}$ The strength of this perturbation can be evaluated from the tunnel splitting $\Delta_{7,-8}$, obtained by applying the Landau-Zener model ${ }^{24}$ to the observed magnetization jump. We find $\Delta_{7,-8}=8.5 \times 10^{-8} \mathrm{meV}$, which is more than 3 orders of magnitude smaller than the $\Delta_{-6,6}=3.2 \times 10^{-4} \mathrm{meV}$ splitting at $B=0 \mathrm{~T}$ due to the $C_{6 v} \mathrm{CF}$, obtained by multiplet calculations. Finally, we ascribe the small jump in the magnetization at $B=5.6 \mathrm{~T}$ to the crossing between the $J_{z}=+7$ and $J_{z}=-6$ states. This crossing allows QTM mediated by first-order electron or phonon scattering in the $C_{6 v}$ $\mathrm{CF}{ }^{20}$ Figure $3 \mathrm{c}$ illustrates these three different mechanisms.

The observed magnetic hysteresis up to $B= \pm 5.6 \mathrm{~T}$ outperforms the best Dy-based single-ion molecular magnets. ${ }^{5,25}$ This is the consequence of the Dy electronic state combined with the peculiar properties of graphene/ $\operatorname{Ir}(111)$, which efficiently protects the Dy spin from the destabilizing interactions specified in the following. First, from a general point of view, the higher the CF symmetry, the lower the number of channels for QTM. ${ }^{20}$ In the limit of perpendicular anisotropy and $C_{\infty v}$ symmetry, QTM is forbidden and the magnetization of the atom has to overcome the entire MAE barrier to reverse its direction. In this respect, graphene, with its $C_{6 v}$ symmetry, represents an ideal substrate to minimize the possibility of direct QTM. Second, only very few vibrational modes exist for an atom adsorbed on a surface, ${ }^{13}$ thus the energy and momentum exchange with the substrate phonon bath is strongly limited. This reduced coupling is particularly true for graphene, which is the material with the highest stiffness, implying a very small phonon density of states in the few $\mathrm{meV}$ energy range of interest. ${ }^{26}$ In addition, the Ir 

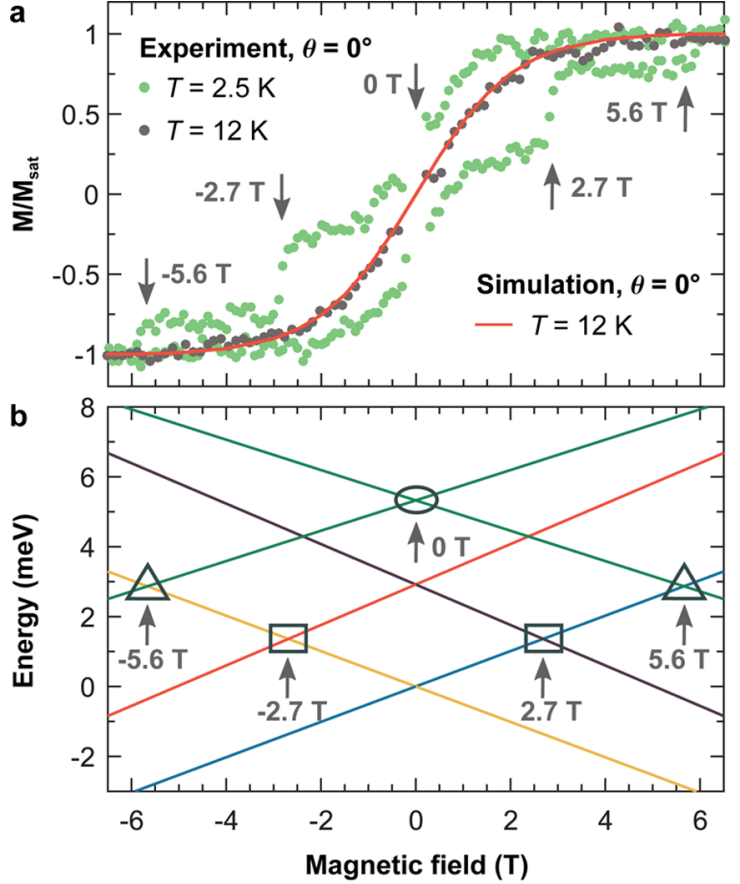

C

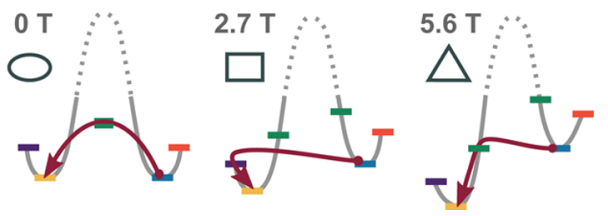

Figure 3. Magnetic level crossing. (a) Magnetization curves at $T=2.5$ $\mathrm{K}$ (green) and $T=12 \mathrm{~K}$ (dark gray) $\left(\phi=5 \phi_{0}, \dot{B}=33 \mathrm{mT} \mathrm{s}^{-1}\right)$. The simulated curve (red) shows magnetization in thermodynamic equilibrium. The arrows point at the steps observed in the magnetization curve acquired at $2.5 \mathrm{~K}$. (b) Zeeman diagram of the lowest lying Dy magnetic levels between -6.8 and $6.8 \mathrm{~T}$. The colors follow the color code of Figure 2c: yellow, $J_{z}=-7$; blue, $J_{z}=7$; purple, $J_{z}=-8$; red, $J_{z}=8$; green, $J_{z}= \pm 6$. The triangles mark crossings of \pm 7 and $\mp 6$ levels at $\pm 5.6 \mathrm{~T}$, rectangles mark crossings of \pm 7 and $\mp 8$ levels at $\pm 2.7 \mathrm{~T}$ and ellipse marks crossing of \pm 6 levels at $0 \mathrm{~T}$. (c) Schematic representation of the relaxation mechanisms; left, thermal excitation to the $J_{z}= \pm 6$ mixed doublet in the $C_{6 v}$ CF; middle, QTM between the $J_{z}$ $= \pm 7$ and the $J_{z}=\mp 8$ crossing states induced by the $C_{3 v} \mathrm{CF}$ perturbation; right, QTM mediated by spin-electron or spin phonon scattering.

vibrational modes are likely filtered by the graphene layer due to its weak van der Waals coupling to the metallic substrate and by the gap of about $6-7 \mathrm{meV}$ in the graphene acoustic out-ofplane phonon modes. ${ }^{26,27}$ Thus, at low temperature, spinphonon coupling is strongly suppressed. Third, for graphene/ $\operatorname{Ir}(111)$, the Dirac point is close to the Fermi level, $E_{\mathrm{F}}$, and there is evidence for the opening of an energy gap around $E_{\mathrm{F}}{ }^{28}$ This suggests very small, if not zero, electron density at $E_{\mathrm{F}}$. Moreover, the weak interaction with the $\operatorname{Ir}(111)$ substrate pushes the graphene to a mean distance of $3.4 \AA$ from the $\operatorname{Ir}(111)$ surface, $^{29}$ hence limiting transmission of the conduction electrons from the Ir substrate through the graphene layer. These three factors altogether lead to strongly reduced spin-flip events.

Missing one of the above-mentioned ingredients is enough to enable fast magnetic relaxation. Increasing the substrate electron density by putting Dy directly on the metallic $\operatorname{Ir}(111)$ surface results in purely paramagnetic magnetization curves (Figure 4a), as also reported for other rare earth atoms on metal surfaces. ${ }^{30}$ Similar arguments hold for Dy atoms on the strongly hybridized graphene $/ \mathrm{Ru}(0001)$, see Figure $4 \mathrm{~b}$. A paramagnetic behavior is again observed for Dy atoms adsorbed on hexagonal boron nitride $(h-\mathrm{BN})$ grown on $\operatorname{Ir}(111)$ (Figure $4 c$ ), in spite of the same $4 \mathrm{f}^{10}$ occupancy and the wide band gap of $h$-BN $(6 \mathrm{eV})$. Here, the advantage of a superior electronic screening with respect to graphene is outweighted by the reduced CF symmetry $\left(C_{3 v}\right)$ of $h-\mathrm{BN}$, resulting in a split doublet as magnetic ground state, for which QTM is strongly activated (see Supporting Information).

Given the single atomic layer thickness of graphene, screening against electron and phonon scattering is effective only at very low temperatures. At $12 \mathrm{~K}$, we observe a perfectly paramagnetic magnetization curve (Figure 3a), indicating that the lifetime is of the order of seconds or less. As demonstrated for $\mathrm{Ho}$ atoms on $\mathrm{MgO} / \mathrm{Ag}(100),{ }^{13}$ stronger protection can be achieved by increasing the number of decoupling layers. Thus, higher thermal stability of the Dy single atom magnet superlattice can possibly be achieved by growing graphene on insulating substrates.

The magnetic states of the Dy atoms in the superlattice are expected to be read and written analogously to Ho atoms on $\mathrm{MgO},{ }^{14}$ thus pioneering the principle of single atom magnetic recording.

Methods. Sample Preparation. The $\operatorname{Ir}(111)$ and $\mathrm{Ru}(0001)$ single crystals were prepared in situ by repeated cycles of $\mathrm{Ar}^{+}$ sputtering and annealing. Subsequently, graphene and $h-\mathrm{BN}$ were grown by chemical vapor deposition using ethylene (100 Langmuir at $1400 \mathrm{~K}$ ) and borazine (125 Langmuir at $1030 \mathrm{~K}$ ), respectively. Both reactions are self-limited to one monolayer since the catalytic dissociation of the respective precursor molecule requires bare metal areas. Dy was deposited from a high purity rod $(99.9 \%)$ using an e-beam evaporator in a background pressure of $\leq 3 \times 10^{-11} \mathrm{mbar}$.

$X$-ray Absorption Spectroscopy. The X-ray absorption measurements were performed at the EPFL/PSI X-Treme beamline at the Swiss Light Source, Paul Scherrer Institut, Villigen, Switzerland. ${ }^{31}$ The experiments were carried out in the TEY mode at $2.5 \mathrm{~K}$, for circularly $\left(\sigma^{+}, \sigma^{-}\right)$and linearly $\left(\sigma^{h}, \sigma^{\nu}\right)$ polarized X-rays, with the magnetic field applied parallel to the incident X-ray beam. The XAS corresponds to $\left(\sigma^{+}+\sigma^{-}\right)$, while the XMCD and XMLD correspond to $\left(\sigma^{+}-\sigma^{-}\right)$and $\left(\sigma^{h}-\sigma^{\nu}\right)$, respectively. The TEY mode enables the high sensitivity required by the extremely low concentration of magnetic elements at the surface. The XAS spectra were acquired with the magnetic field collinear with the photon beam at normal $(\theta$ $=0^{\circ}$ ) and grazing incidence $\left(\theta=60^{\circ}\right)$. To take into account the different surface areas illuminated by the X-ray beam at both sample orientations, the XAS spectra were normalized to the intensity at the pre-edge $(1281 \mathrm{eV})$. Prior to deposition of Dy, background spectra on the given substrate have been recorded and then subtracted from the Dy XAS spectra to eliminate any contribution from the substrate. The absolute value of the X-ray flux was measured with a photodiode placed after the last optical element of the beamline and is given in units of $\phi_{0}=3$ $\times 10^{-3}$ photons $\mathrm{nm}^{-2} \mathrm{~s}^{-1}$.

Scanning Tunneling Microscopy. The STM measurements were performed with a homemade low temperature STM, operating at $5 \mathrm{~K}^{32}$ We used $\mathrm{W}$ tips for all measurements. The STM images were acquired in constant current mode. The bias voltages given in the figure captions refer to the sample; 

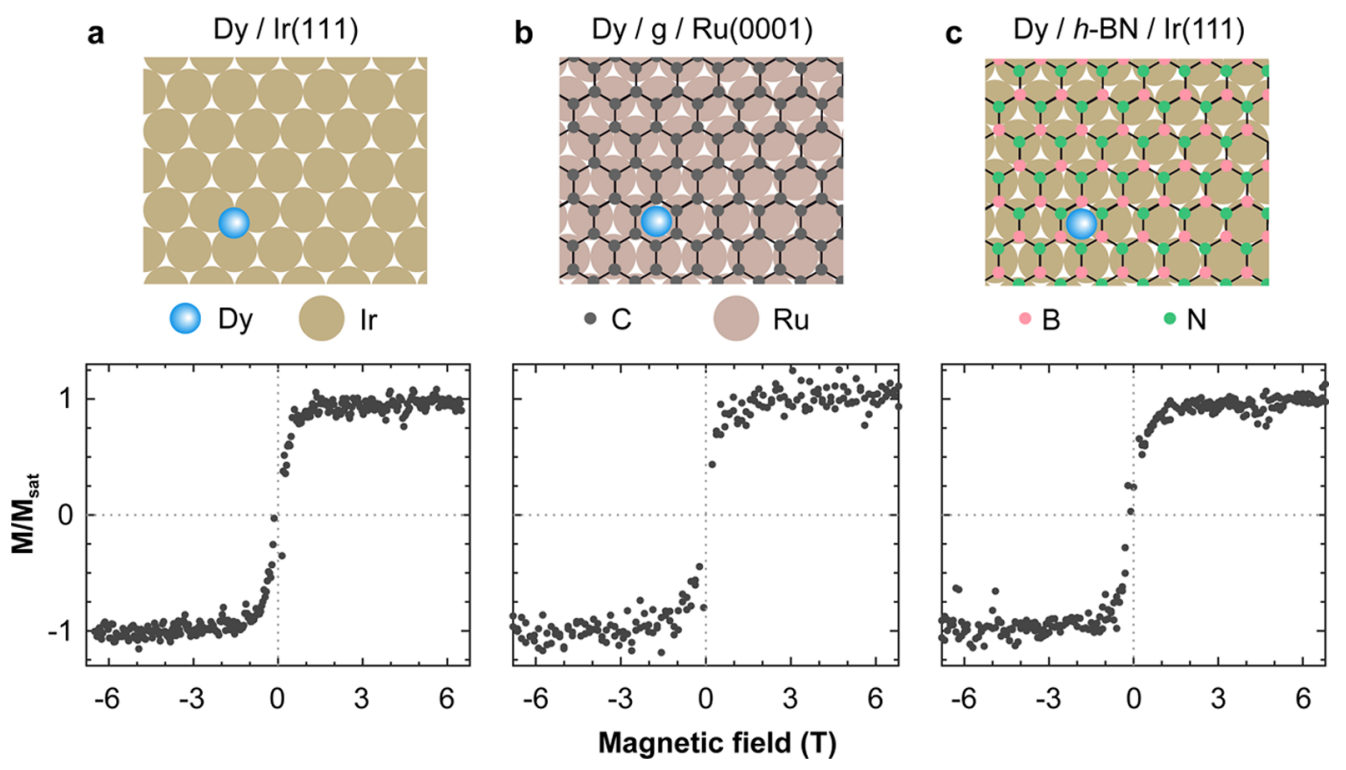

Figure 4. Paramagnetic behavior of Dy on other substrates. Dy atoms on (a) $\operatorname{Ir}(111)$, (b) graphene $/ \operatorname{Ru}(0001)$, and (c) $h$-BN/Ir(111). Top, schematic drawing of adsorption geometry; bottom, experimental magnetization curves $\left(T_{\text {dep }}=4 \mathrm{~K}, T=2.5 \mathrm{~K}, \theta=0^{\circ}\right.$; (a) $\phi=9 \phi_{0}, \dot{B}=12 \mathrm{mT} \mathrm{s}^{-1}$; (b,c) $\phi=5 \phi_{0}, \dot{B}=33 \mathrm{mT} \mathrm{s}^{-1}$ ).

therefore, at the used negative values, electrons tunnel from occupied states of the sample into empty states of the tip.

\section{ASSOCIATED CONTENT}

\section{S Supporting Information}

The Supporting Information is available free of charge on the ACS Publications website at DOI: 10.1021/acs.nanolett.6b03543.

Description of the Dy adsorption site, of the selfassembly on graphene/ $\operatorname{Ir}(111)$, details on the multiplet calculations of Dy on graphene/ $\operatorname{Ir}(111)$ and $h$-BN/ $\operatorname{Ir}(111)$, as well as XAS, XMCD, and XMLD spectra of Dy on $\operatorname{Ir}(111)$ and graphene $/ \mathrm{Ru}(0001)$ (PDF)

Ligand input file for multiX code, used in calculations of spectra for Dy on graphene/(111) (TXT)

Ligand input file for multiX code, used in calculations of spectra for Dy on $h$-BN/Ir(111) (TXT)

\section{AUTHOR INFORMATION}

\section{Corresponding Author}

*E-mail: harald.brune@epfl.ch.

Notes

The authors declare no competing financial interest.

\section{ACKNOWLEDGMENTS}

We gratefully acknowledge funding by the Swiss National Science Foundation (Grants 200021 146715/1, PZ00P2_142474 and 200020_157081/1). We further acknowledge A. Uldry and B. Delley for providing us with the updated version of the multiX software. We thank K. Diller for technical support and fruitful discussions.

\section{REFERENCES}

(1) Bennewitz, R.; Crain, J. N.; Kirakosian, A.; Lin, J.-L.; McChesney, J. L.; Petrovykh, D. Y.; Himpsel, F. J. Atomic Scale Memory at a Silicon Surface. Nanotechnology 2002, 13, 499.

(2) Kalff, F. E.; Rebergen, M. P.; Fahrenfort, E.; Girovsky, J.; Toskovic, R.; Lado, J. L.; Fernández-Rossier, J.; Otte, A. F. A Kilobyte
Rewritable Atomic Memory. Nat. Nanotechnol. 2016, DOI: 10.1038/ nnano.2016.131.

(3) Komeda, T.; Isshiki, H.; Liu, J.; Zhang, Y.-F.; Lorente, N.; Katoh, K.; Breedlove, B. K.; Yamashita, M. Observation and Electric Current Control of a Local Spin in a Single-Molecule Magnet. Nat. Commun. 2011, 2, 217.

(4) Gambardella, P.; Stepanow, S.; Dmitriev, A.; Honolka, J.; de Groot, F. M. F.; Lingenfelder, M.; Gupta, S. S.; Sarma, D. D.; Bencok, P.; Stanescu, S.; et al. Supramolecular Control of the Magnetic Anisotropy in Two-Dimensional High-Spin Fe Arrays at a Metal Interface. Nat. Mater. 2009, 8, 189.

(5) Chen, Y.-C.; Liu, J.-L.; Ungur, L.; Liu, J.; Li, Q.-W.; Wang, L.-F.; Ni, Z.-P.; Chibotaru, L. F.; Chen, X.-M.; Tong, M.-L. SymmetrySupported Magnetic Blocking at $20 \mathrm{~K}$ in Pentagonal Bipyramidal Dy(III) Single-Ion Magnets. J. Am. Chem. Soc. 2016, 138, 2829-2837.

(6) Wäckerlin, C.; Donati, F.; Singha, A.; Baltic, R.; Rusponi, S.; Diller, K.; Patthey, F.; Pivetta, M.; Lan, Y.; Klyatskaya, S.; et al. Giant Hysteresis of Single-Molecule Magnets Adsorbed on a Nonmagnetic Insulator. Adv. Mater. 2016, 28, 5195-5199.

(7) Gambardella, P.; Rusponi, S.; Veronese, M.; Dhesi, S. S.; Grazioli, C.; Dallmeyer, A.; Cabria, I.; Zeller, R.; Dederichs, P. H.; Kern, K.; et al. Giant Magnetic Anisotropy of Single Cobalt Atoms and Nanoparticles. Science 2003, 300, 1130-1133.

(8) Hirjibehedin, C. F.; Lin, C.-Y.; Otte, A. F.; Ternes, M.; Lutz, C. P.; Jones, B. A.; Heinrich, A. J. Large Magnetic Anisotropy of a Single Atomic Spin Embedded in a Surface Molecular Network. Science 2007, 317, 1199-1203.

(9) Meier, F.; Zhou, L.; Wiebe, J.; Wiesendanger, R. Revealing Magnetic Interactions from Single-Atom Magnetization Curves. Science 2008, 320, 82-86.

(10) Khajetoorians, A. A.; Lounis, S.; Chilian, B.; Costa, A. T.; Zhou, L.; Mills, D. L.; Wiebe, J.; Wiesendanger, R. Itinerant Nature of AtomMagnetization Excitation by Tunneling Electrons. Phys. Rev. Lett. 2011, 106, 037205.

(11) Donati, F.; Dubout, Q.; Autès, G.; Patthey, F.; Calleja, F.; Gambardella, P.; Yazyev, O. V.; Brune, H. Magnetic Moment and Anisotropy of Individual Co Atoms on Graphene. Phys. Rev. Lett. 2013, 111, 236801.

(12) Rau, I. G.; Baumann, S.; Rusponi, S.; Donati, F.; Stepanow, S.; Gragnaniello, L.; Dreiser, J.; Piamonteze, C.; Nolting, F.; Gangopadhyay, S.; et al. Reaching the Magnetic Anisotropy Limit of a 3d Metal Atom. Science 2014, 344, 988-992. 
(13) Donati, F.; Rusponi, S.; Stepanow, S.; Wäckerlin, C.; Singha, A.; Persichetti, L.; Baltic, R.; Diller, K.; Patthey, F.; Fernandes, E.; et al. Magnetic Remanence in Single Atoms. Science 2016, 352, 318-321.

(14) Natterer, F. D.; Yang, K.; Paul, W.; Choi, T.; Greber, T.; Heinrich, A.; Lutz, C. P. Reading and Writing Single-Atom Magnets, 2016; https://arxiv.org/abs/1607.03977.

(15) N’Diaye, A. T.; Bleikamp, S.; Feibelman, P. J.; Michely, T. TwoDimensional Ir Cluster Lattice on a Graphene Moiré on $\operatorname{Ir}(111)$. Phys. Rev. Lett. 2006, 97, 215501.

(16) Thomas, L.; Lionti, F.; Ballou, R.; Gatteschi, D.; Sessoli, R.; Barbara, B. Macroscopic Quantum Tunnelling of Magnetization in a Single Crystal of Nanomagnets. Nature 1996, 383, 145.

(17) Dreiser, J.; Westerström, R.; Piamonteze, C.; Nolting, F.; Rusponi, S.; Brune, H.; Yang, S.; Popov, A.; Dunsch, L.; Greber, T. Xray Induced Demagnetization of Single-Molecule Magnets. Appl. Phys. Lett. 2014, 105, 032411.

(18) Uldry, A.; Vernay, F.; Delley, B. Systematic Computation of Crystal-Field Multiplets for X-ray Core Spectroscopies. Phys. Rev. B: Condens. Matter Mater. Phys. 2012, 85, 125133.

(19) Miyamachi, T.; Schuh, T.; Märkl, T.; Bresch, C.; Balashov, T.; Stöhr, A.; Karlewski, C.; André, S.; Marthaler, M.; Hoffmann, M.; et al. Stabilizing the Magnetic Moment of Single Holmium Atoms by Symmetry. Nature 2013, 503, 242-246.

(20) Hübner, C.; Baxevanis, B.; Khajetoorians, A. A.; Pfannkuche, D. Symmetry Effects on the Spin Switching of Adatoms. Phys. Rev. B: Condens. Matter Mater. Phys. 2014, 90, 155134.

(21) Ishikawa, N.; Sugita, M.; Wernsdorfer, W. Quantum Tunneling of Magnetization in Lanthanide Single-Molecule Magnets: Bis(phthalocyaninato)terbium and Bis(phthalocyaninato)dysprosium Anions. Angew. Chem., Int. Ed. 2005, 44, 2931-2935.

(22) Uchoa, B.; Yang, L.; Tsai, S.-W.; Peres, N. M. R.; Castro Neto, A. H. Orbital Symmetry Fingerprints for Magnetic Adatoms in Graphene. New J. Phys. 2014, 16, 013045.

(23) Cervetti, C.; Rettori, A.; Pini, M. G.; Cornia, A.; Repollés, A.; Luis, F.; Dressel, M.; Rauschenbach, S.; Kern, K.; Burghard, M.; et al. The Classical and Quantum Dynamics of Molecular Spins on Graphene. Nat. Mater. 2016, 15, 164.

(24) Wernsdorfer, W.; Sessoli, R.; Caneschi, A.; Gatteschi, D.; Cornia, A. Nonadiabatic Landau-Zener Tunneling in $\mathrm{Fe}_{8}$ Molecular Nanomagnets. Europhys. Lett. 2000, 50, 552.

(25) Westerström, R.; Dreiser, J.; Piamonteze, C.; Muntwiler, M.; Weyeneth, S.; Brune, H.; Rusponi, S.; Nolting, F.; Popov, A.; Yang, S.; et al. An Endohedral Single-Molecule Magnet with Long Relaxation Times: $\mathrm{DySc}_{2} \mathrm{~N} @ \mathrm{C}_{80}$. J. Am. Chem. Soc. 2012, 134, 9840.

(26) Endlich, M.; Molina-Sánchez, A.; Wirtz, L.; Kröger, J. Screening of Electron-Phonon Coupling in Graphene on $\operatorname{Ir}(111)$. Phys. Rev. B: Condens. Matter Mater. Phys. 2013, 88, 205403.

(27) Chen, L.; Huang, Z.; Kumar, S. Phonon Transmission and Thermal Conductance Across Graphene/Cu Interface. Appl. Phys. Lett. 2013, 103, 123110.

(28) Rusponi, S.; Papagno, M.; Moras, P.; Vlaic, S.; Etzkorn, M.; Sheverdyaeva, P. M.; Pacilé, D.; Brune, H.; Carbone, C. Highly Anisotropic Dirac Cones in Epitaxial Graphene Modulated by an Island Superlattice. Phys. Rev. Lett. 2010, 105, 246803.

(29) Hämäläinen, S. K.; Boneschanscher, M. P.; Jacobse, P. H.; Swart, I.; Pussi, K.; Moritz, W.; Lahtinen, J.; Liljeroth, P.; Sainio, J. Structure and Local Variations of the Graphene Moiré on Ir(111). Phys. Rev. B: Condens. Matter Mater. Phys. 2013, 88, 201406.

(30) Donati, F.; Singha, A.; Stepanow, S.; Wäckerlin, C.; Dreiser, J.; Gambardella, P.; Rusponi, S.; Brune, H. Magnetism of Ho and Er Atoms on Close-Packed Metal Surfaces. Phys. Rev. Lett. 2014, 113, 237201.

(31) Piamonteze, C.; Flechsig, U.; Rusponi, S.; Dreiser, J.; Heidler, J.; Schmidt, M.; Wetter, R.; Calvi, M.; Schmidt, T.; Pruchova, H.; et al. XTreme Beamline at SLS: X-ray Magnetic Circular and Linear Dichroism at High Field and Low Temperature. J. Synchrotron Radiat. 2012, 19, 661-674.
(32) Gaisch, R.; Gimzewski, J. K.; Reihl, B.; Schlittler, R. R.; Tschudy, M.; Schneider, W. D. Low-Temperature Ultra-High Vacuum Scanning Tunneling Microscope. Ultramicroscopy 1992, 42-44, 1621. 


\section{Supporting Information for "Superlattice of}

\section{Single Atom Magnets on Graphene"}

Romana Baltic, $^{\dagger}$ Marina Pivetta, $^{\dagger}$ Fabio Donati, $^{\dagger}$ Christian Wäckerlin $^{\dagger}$ Aparajita Singha, ${ }^{\dagger}$ Jan Dreiser ${ }^{\dagger, \ddagger}$ Stefano Rusponi, ${ }^{\dagger}$ and Harald Brune ${ }^{*, \dagger}$

Institute of Physics, Ecole Polytechnique Fédérale de Lausanne, Station 3, CH-1015

Lausanne, Switzerland, and Swiss Light Source, Paul Scherrer Institute, CH-5232 Villigen PSI, Switzerland

E-mail: harald.brune@epfl.ch

${ }^{*}$ To whom correspondence should be addressed

†Institute of Physics, Ecole Polytechnique Fédérale de Lausanne, Station 3, CH-1015 Lausanne, Switzerland

${ }^{\ddagger}$ Swiss Light Source, Paul Scherrer Institute, CH-5232 Villigen PSI, Switzerland 


\section{Contents}

1 Self-assembly of Dy superlattice on graphene/Ir(111) 3

2 Adsorption site of Dy on graphene/Ir(111) 5

3 Additional XAS and XMCD data $\quad 6$

4 Multiplet calculations: Dy on graphene/Ir(111) and $h$-BN/Ir(111) 8

5 Dy on $\operatorname{Ir}(111)$ and on graphene/Ru(0001) 11

6 Bibliography 12 


\section{Self-assembly of Dy superlattice on graphene/Ir(111)}

The scanning tunneling microscopy (STM) image in Figure S1a shows an ensemble of Dy atoms on graphene/Ir(111) after deposition of $0.01 \mathrm{ML}$ Dy at $40 \mathrm{~K}$ (as pointed out in the main paper, the monolayer (ML) definition is one Dy atom per graphene unit cell). At this substrate temperature, adatom diffusion is activated. The potential landscape seen by the diffusing Dy atoms has three components: a) the atomic corrugation of the graphene lattice, b) the long period surface potential due to the graphene moiré, and c) the Coulomb repulsion amongst the atoms generated by the strong charge transfer from the Dy to the graphene. ${ }^{1,2}$ Similar to Cs atoms on graphene/6H-SiC(0001), ${ }^{3}$ the observed superlattice results from the
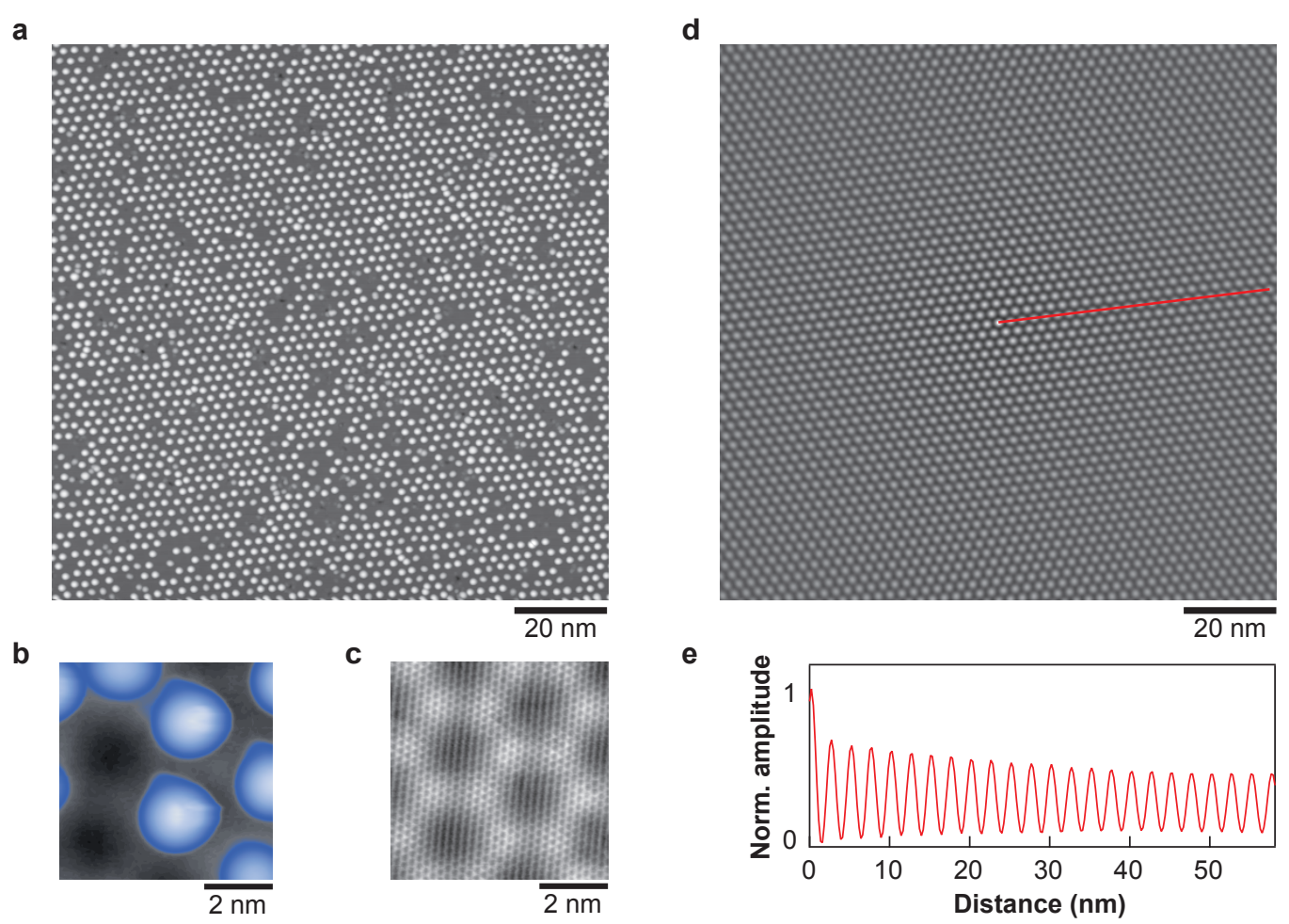

Figure S1. Long range order of the Dy superlattice. (a) STM image of Dy superlattice on graphene $/ \operatorname{Ir}(111)\left(V_{\mathrm{t}}=-0.2 \mathrm{~V}, I_{\mathrm{t}}=100 \mathrm{pA} ; T_{\text {dep }}=40 \mathrm{~K}, T=5 \mathrm{~K}\right)$. (b) Detail of the Dy superlattice in (a) $\left(V_{\mathrm{t}}=-0.4 \mathrm{~V}, I_{\mathrm{t}}=20 \mathrm{pA} ; T=5 \mathrm{~K}\right)$. (c) Atomically resolved STM image of the graphene moiré structure: the atop-regions appear as dark areas. By comparing (b) and (c) we identify the atop-position as the stable adsorption position for the Dy monomers $\left(V_{\mathrm{t}}=-0.02 \mathrm{~V}, I_{\mathrm{t}}=50 \mathrm{pA} ; T=5 \mathrm{~K}\right)$. (d) Autocorrelation function of the image shown in (a). (e) Line cut along the red line shown in (d). 
cooperative effect of the latter two components, with the Coulomb repulsion preventing dimer formation upon diffusion, and the moiré potential defining the array of stable adsorption sites. The equilibrium positions correspond to Dy occupying the atop stacking region of the moiré pattern, i.e., where the $\mathrm{C}_{6}$ ring of graphene is centered above an Ir substrate atom. The apparent height of the atop, fcc and hcp stacking areas depends on the tunneling conditions. ${ }^{4}$ In Figure S1b the atop regions appear dark. Figure S1c shows the atomically resolved graphene structure where one recognizes the atop-region (dark) with the fcc and hcp regions appearing bright. ${ }^{4}$ We note that the Dy atom superlattice forms at $30 \mathrm{~K} \leq T_{\text {dep }} \leq 50 \mathrm{~K}$. For $T_{\text {dep }}<30 \mathrm{~K}$ the inter-moiré cell diffusion is suppressed, resulting in disordered arrangement of adatoms. For $T_{\text {dep }}>50 \mathrm{~K}$ the diffusing adatoms can overcome the repulsive Coulomb barrier and dimers or bigger clusters form. The long range order of the Dy atom superlattice is highlighted by the autocorrelation function ${ }^{5}$ whose intensity shows a very shallow decay with distance (Figure S1d,e).

A detailed analysis of both ordered and disordered Dy samples reveals the presence of two kinds of protrusions characterized by different apparent heights (see Figure S2a). From the line profiles shown in Figure S2c we measure $0.50 \pm 0.02 \mathrm{~nm}$ (species A) and $0.41 \pm 0.02 \mathrm{~nm}$ (species B). The absolute heights depend on the bias $V_{\mathrm{t}}$, however, species A always appears significantly higher. To identify the nature of these two species we performed manipulations by centering the STM tip on top of them and applying a tunnel voltage ramp. Type A is laterally displaced for tunnel voltages exceeding $V_{\mathrm{t}}= \pm 2.5 \mathrm{~V}$, while type B is split into two objects of type A for tunnel voltages exceeding $V_{\mathrm{t}}= \pm 1.5 \mathrm{~V}$. The arrow in Figure S2a points to the object of type B before the manipulation, and arrows in Figure S2b point to the two objects of type A emerging after the splitting. We therefore identify species A as monomers and species B as dimers. Figure S2d shows the difference between Figures S2a and b, with the split dimer appearing in black, and the two newly emerged monomers in white. For a Dy coverage of $0.01 \mathrm{ML}$, a statistical analysis performed over hundreds of protrusions reveals that $83 \pm 3 \%$ of them are monomers and $17 \pm 3 \%$ are dimers both for $T_{\text {dep }}=10 \mathrm{~K}$ and 
a

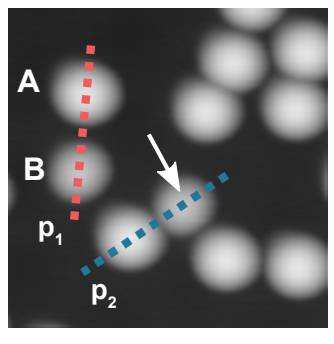

C

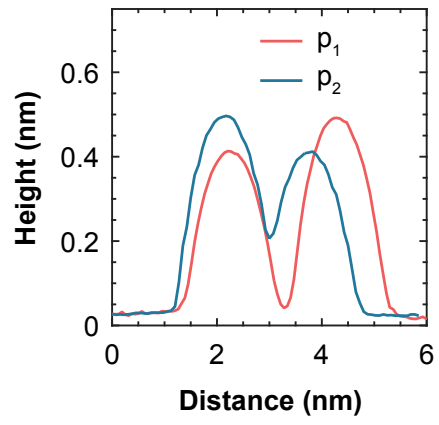

b
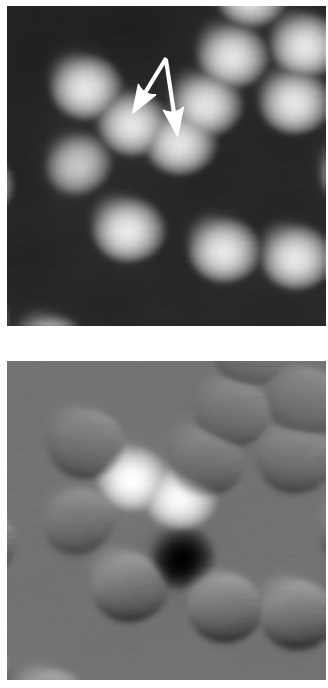

Figure S2. Dy monomers and dimers. (a,b) Sequence of STM images showing the splitting of a dimer into two monomers by manipulations with the STM tip. The arrow on image (a) points at the dimer before the splitting, and arrows on image (b) point at the two monomers resulting from the split dimer $\left(V_{\mathrm{t}}=-0.5 \mathrm{~V}, I_{\mathrm{t}}=20 \mathrm{pA} ; 8.3 \times 8.3 \mathrm{~nm}^{2}, T_{\text {dep }}=10 \mathrm{~K}\right.$, $T=5 \mathrm{~K}$ ). (c) Two STM profiles, indicated by dashed lines on image (a), show apparent heights of monomers (A) and dimers (B). Both profiles start at the bottom left of the image and have following sequences: dimer-monomer in red, monomer-dimer in blue. (d) The difference between images (a) and (b), showing the splitting of dimer (black) into two monomers (white).

$T_{\text {dep }}=40 \mathrm{~K}$. The contribution of these two species to the XAS and XMCD spectra are discussed in Section 3.

\section{Adsorption site of Dy on graphene/Ir(111)}

The adsorption site of an atom on the surface determines the symmetry and strength of the crystal field. We performed STM measurements to experimentally determine the adsorption site of Dy atoms on graphene/Ir(111). Figure S3 shows an atomically resolved STM image of graphene with a Dy atom after deposition at $10 \mathrm{~K}$. Black lines in the figure mark the sixfold graphene hollow sites, i.e., the centers of the hexagonal carbon rings, in the vicinity of the adsorbed Dy. Their intersection occurs at the summit of the Dy atom, thus 


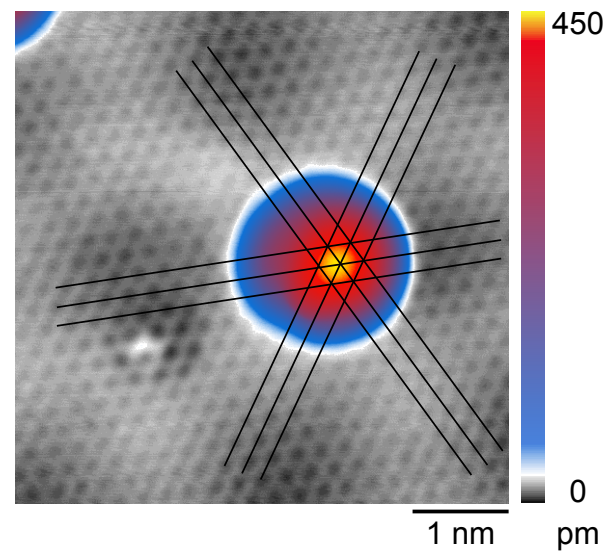

Figure S3. Adsorption site of Dy atoms on graphene/Ir(111). Atomically resolved STM image of graphene/ $\operatorname{Ir}(111)$ with an adsorbed Dy atom. Black lines mark the hollow sites of the graphene lattice $\left(V_{\mathrm{t}}=-0.05 \mathrm{~V}, I_{\mathrm{t}}=500 \mathrm{pA} ; T_{\mathrm{dep}}=10 \mathrm{~K}, T=5 \mathrm{~K}\right)$.

identifying the hollow site as the adsorption site of Dy on graphene/ $\operatorname{Ir}(111)$.

\section{Additional XAS and XMCD data}

In order to address the effect of dimers and bigger clusters on the magnetic properties of an ensemble of Dy monomers on graphene/Ir(111), we performed coverage dependent measurements. As shown in Figure S4, XAS and XMCD spectra exhibit, in addition to the
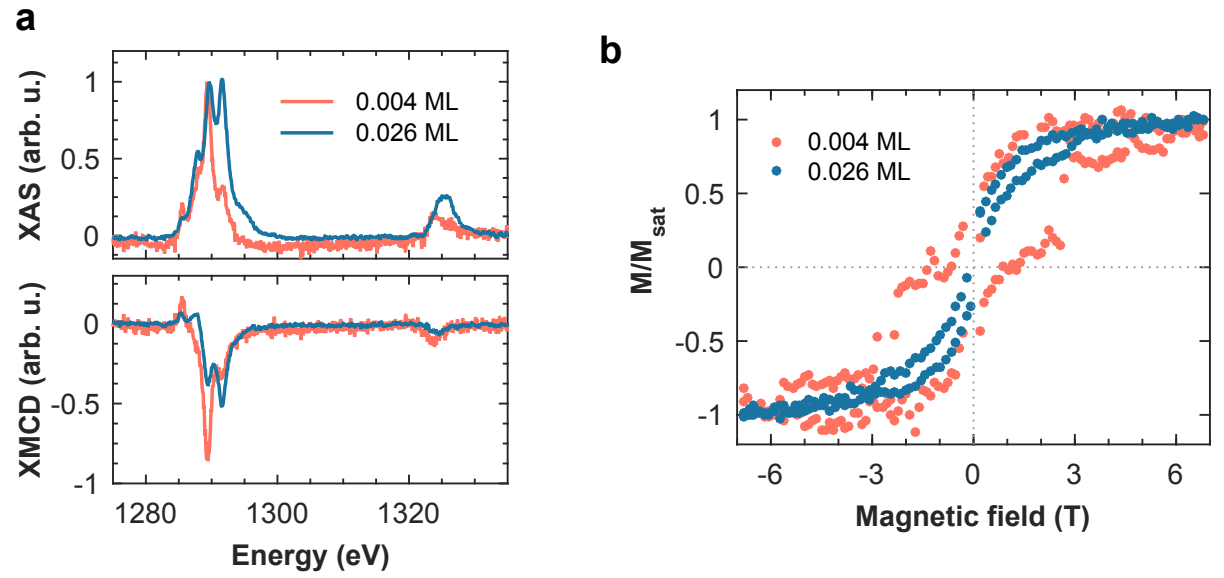

Figure S4. Coverage dependence of absorption spectra and magnetization curves. (a) XAS and XMCD spectra, and (b) magnetization curve for 0.004 ML (red) and 0.026 ML (blue) of Dy $\left(T=2.5 \mathrm{~K}\right.$; (a) $B=6.8 \mathrm{~T}$, (b) $\left.\phi=5 \phi_{0}, \dot{B}=33 \mathrm{mT} \mathrm{s}^{-1}\right)$. 
main peak at $1289.3 \mathrm{eV}$, a peak at $1291.8 \mathrm{eV}$ whose intensity increases with the Dy coverage increasing from 0.004 ML to 0.026 ML. This peak is absent in the simulated spectra of monomers (see Figure 2) and originates from dimers and bigger clusters formed on the surface. Its energy is due to a change in the orbital occupancy from $4 f^{10}$ to the bulk value of $4 f^{9} \cdot{ }^{6}$ Thus, recording the XMCD signal at $1289.3 \mathrm{eV}$ or $1291.8 \mathrm{eV}$ allows to separately address the magnetic behavior of monomers and clusters, respectively.

Figure S4b shows magnetization curves acquired at the XMCD monomer peak (1289.3 eV) for $0.004 \mathrm{ML}$ and 0.026 ML of Dy. At $0.026 \mathrm{ML}$, we observe a narrowing of the hysteresis indicating that the magnetic interaction with neighboring atoms/clusters shortens the magnetic lifetime of monomers. On the contrary, below $0.01 \mathrm{ML}$ the shape of the magnetization curve and the magnetic lifetime are coverage independent.

As it can be seen from Figure S5b, the magnetization curve acquired at grazing incidence $\left(\theta=60^{\circ}\right)$ also exhibits magnetic remanence and hysteresis up to about $6 \mathrm{~T}$. At $\theta=60^{\circ}$ the out-of-plane component of the magnetic field is reduced by a factor of two. Therefore,
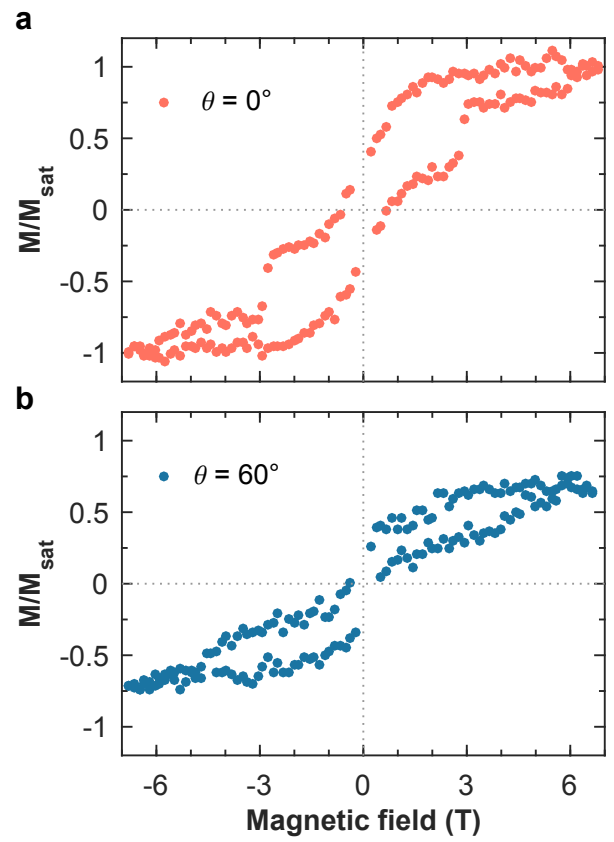

Figure S5. Angular dependence of Dy magnetization curve: (a) $\theta=0^{\circ}$ and (b) $\theta=60^{\circ}$ $\left(T=2.5 \mathrm{~K}, \phi=5 \phi_{0}, \dot{B}=33 \mathrm{mT} \mathrm{s}^{-1}\right)$. 
simply considering its geometrical projection, the values at which QTM steps occur should be shifted to twice the field values found for $\theta=0^{\circ}$ (Figure S5a). Specifically, the QTM step found at $2.7 \mathrm{~T}$ in normal incidence should shift to $5.4 \mathrm{~T}$ when measured at grazing angle. Instead, we observe a broad step at around $4.5 \mathrm{~T}$ in the hysteresis loop at grazing incidence. We ascribe this discrepancy to the presence of a strong transverse field that enhances the electron/phonon mediated relaxation processes and broadens the magnetic field region where QTM occurs. ${ }^{7}$

\section{Multiplet calculations: Dy on graphene/Ir(111) and $h-\mathrm{BN} / \operatorname{Ir}(111)$}

To gain information on the energy spectrum and occupation of the Dy quantum states, we have performed atomic multiplet simulations of the experimentally acquired X-ray absorption spectroscopy (XAS), magnetic circular dichroism (XMCD) and magnetic linear dichroism (XMLD) spectra using the multiX software. ${ }^{8}$ The code uses an effective point charge approach for the description of the crystal field generated by the interaction of the Dy atom with the surrounding substrate atoms. This description holds in particular for the $4 f$ states that we probe at the $M_{4,5}$ edges. These states are highly localized which reduces their interaction with the surrounding substrate atoms to a purely electrostatic one. ${ }^{9,10}$ The position and intensity of the effective charges were chosen in order to simultaneously fit the shape and intensity of XAS, XMCD, and XLD spectra. For Dy on graphene/Ir(111) the charge position and sign were inspired by DFT calculations. ${ }^{1,2}$ Figure S6 summarizes the spatial distribution and the values used for the effective charges in the case of Dy on graphene/ $\operatorname{Ir}(111)$ and on $h-\mathrm{BN} / \operatorname{Ir}(111)$. The values of the spin-orbit coupling and Coulomb interactions were scaled to $75 \%$ and $90 \%$ of the Hartree-Fock values, respectively. The experimental line broadening due to the finite lifetime of the core-hole state was modeled by convolution with a Gaussian of $\sigma=0.6 \mathrm{eV}$. Ligand input files for the multiX code de- 


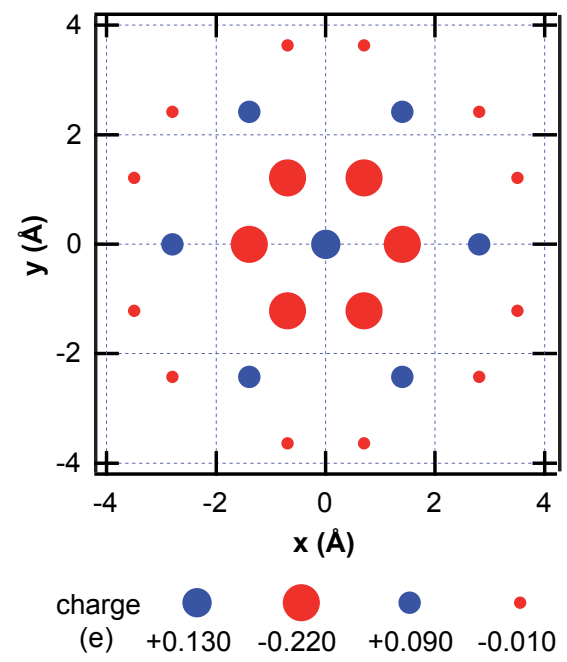

b

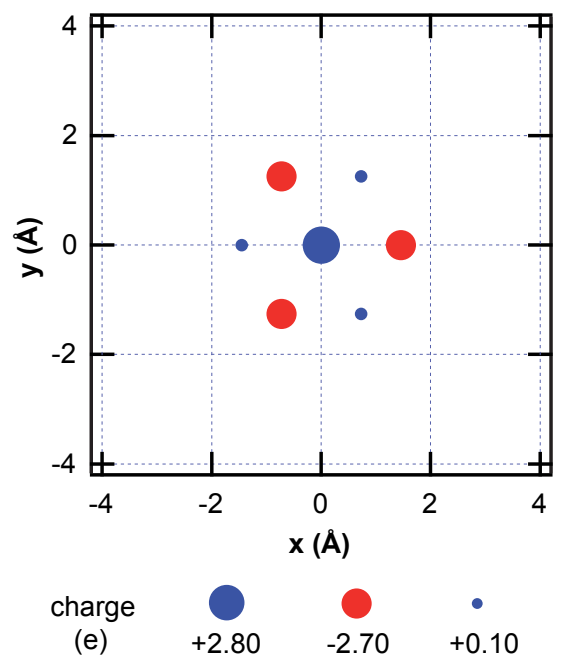

Figure S6. Point charge crystal field. Spatial distribution of point charges for Dy on (a) graphene/ $\operatorname{Ir}(111)$ and (b) on h-BN/Ir(111). For each panel, the size of the circle is proportional to the charge value, and the color represents the sign of the charge (blue = positive, red $=$ negative). This corresponds to a $\mathrm{CF}$ with $C_{6 v}$ symmetry in (a) and with $C_{3 v}$ symmetry in (b).

scribing the list of charges and positions are given as separate files: graphene-CF.txt for graphene/Ir(111), and $h-B N-C F$.txt for $h$-BN $/ \operatorname{Ir}(111)$. In these files, the first line is a multiplication factor required by the code; the following columns, from left to right, represent the $x, y$, and $z$ positions, and the values of the charges with the Dy atom placed at the origin of the coordinate system.

Further constraint for the simulation of Dy on graphene/ $\operatorname{Ir}(111)$ is given by the fielddependent crossings of the magnetic levels, responsible for the steps observed in the magnetization curves, which unambiguously determine the energy splitting of the magnetic levels. Figures 2 and 3 of the main text show an overall good agreement between experimental and simulated spectra, and between the measured magnetization curve and the calculated level splitting, respectively. The spectra differ only in the XAS peak at $1291.8 \mathrm{eV}$ observed in the experiment and absent in the simulation. The origin of this peak are Dy dimers present on the surface (see Sections 1 and 3) which are not taken into account by the multiplet calculations. For the Dy monomer we find that the high spin-orbit coupling characteristic 
of rare earths favours the lowest multiplet with a total moment $J=8$. The crystal field of graphene splits these quantum levels generating a degenerate ground state doublet with $J_{z}= \pm 7$, as discussed in the main text.

For Dy on $h$-BN/Ir(111), the intensity of the XAS peak at $1291.8 \mathrm{eV}$ is slightly higher than for graphene/Ir(111) (Figure S7a), suggesting a larger fraction of dimers in the ensemble, possibly due to the larger mobility of the Dy atoms on $h$-BN compared to the one on graphene. The multiplet calculations were focused on reproducing XAS spectra, XMCD angular dependence, and the larger intensity of XMLD at $50 \mathrm{mT}$ compared to $6.8 \mathrm{~T}$ (Figure S7b). Similar to Dy on graphene/Ir(111), we find 10 electrons occupying the Dy $4 f$
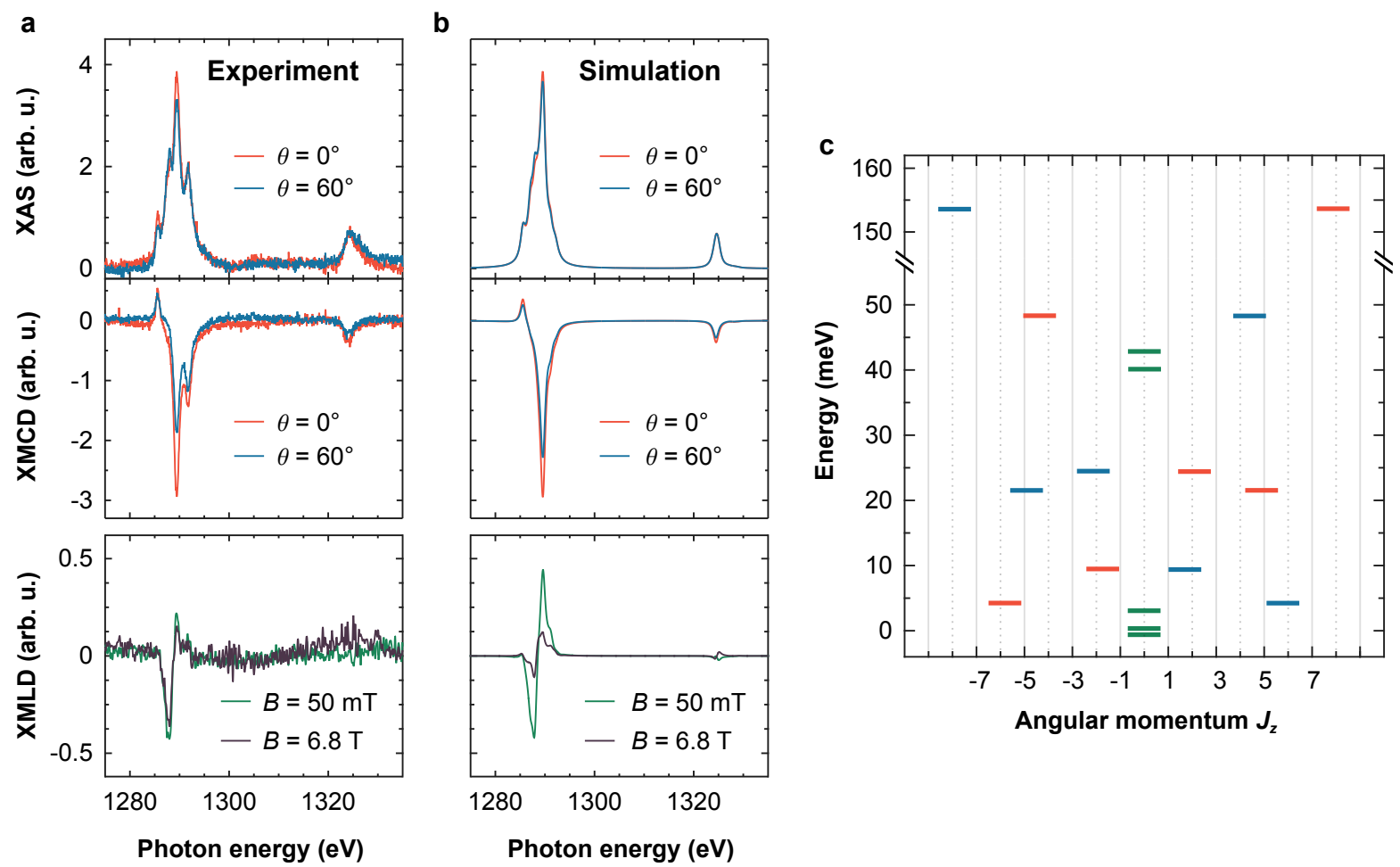

Figure S7. Dy atoms on $h$-BN/Ir(111). (a) Experimentally acquired XAS, XMCD and XMLD spectra at Dy $M_{4,5}$ edge $\left(B=6.8 \mathrm{~T}, T_{\text {dep }}=4 \mathrm{~K}, T=2.5 \mathrm{~K}\right)$. (b) XAS, XMCD and XMLD spectra resulting from multiplet calculations for single Dy atoms in the $C_{3 v} \mathrm{CF}$ of $h$-BN/Ir(111). (c) Splitting of the Dy lowest multiplet $(J=8)$ according to the multiplet calculations. Due to the strong $\mathrm{CF}$, each state is a mixture of states with different $J_{z}$, and the color code identifies magnetic quantum states connected by symmetry operation within the $C_{3 v}$ group. The splitting of the two lowest states with $J_{z}=0$ is emphasized for better clarity. 
orbitals and a lowest multiplet with a total moment $J=8$. However, the magnetic ground state is a singlet originating from a tunnel-split doublet which is not protected from QTM. The strong mixing of the two states is highlighted by the large tunnel splitting of about $0.18 \mathrm{meV}$. The energy spectrum of the lowest multiplet is shown in Figure S7c.

\section{Dy on $\operatorname{Ir}(111)$ and on graphene $/ \mathrm{Ru}(0001)$}

Figure S8a,b show the XAS, XMCD, and XMLD spectra of single Dy atoms on $\operatorname{Ir}(111)$ and on graphene/Ru(0001). For Dy on $\operatorname{Ir}(111)$, the acquired XAS line shape is characteristic of Dy $4 f^{9}$ occupation, ${ }^{6}$ and is clearly different from the one of Dy on graphene/ $\operatorname{Ir}(111)$ shown in Figure 2. In addition, the XMCD is independent of the X-ray incidence angle, indicating negligible magnetic anisotropy. As seen in Figure 4a, the magnetization curve
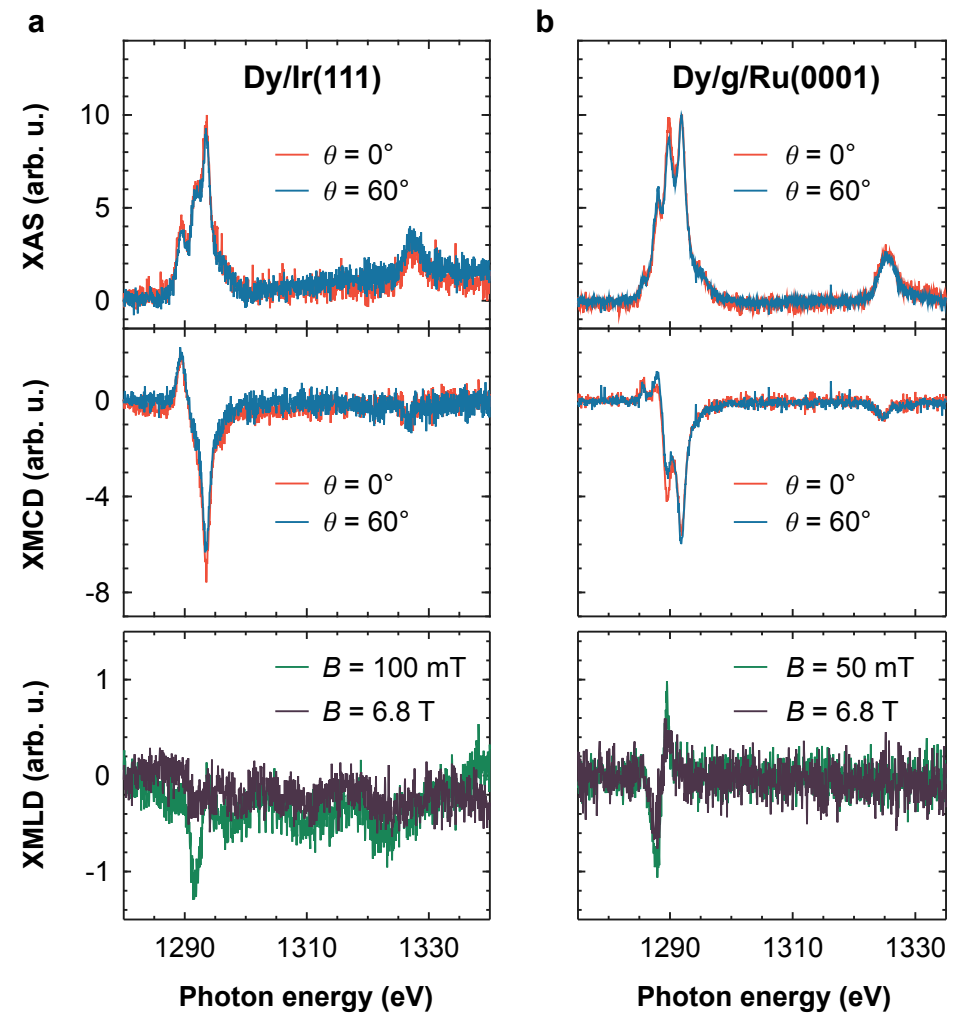

Figure S8. Magnetism of $\mathrm{Dy}$ on $\operatorname{Ir}(111)$ and graphene $/ \mathrm{Ru}(0001)$. Experimentally acquired XAS, XMCD, and XMLD for (a) Dy on $\operatorname{Ir}(111)$ and (b) Dy on graphene $/ \mathrm{Ru}(0001)$ $\left(B=6.8 \mathrm{~T}, T_{\mathrm{dep}}=4 \mathrm{~K}, T=2.5 \mathrm{~K}\right)$. 
saturates for $|B|>1.5 \mathrm{~T}$ and does not show hysteresis, indicating magnetization lifetimes shorter than a few seconds at $2.5 \mathrm{~K}$, a time scale set by the acquisition time of each point of the magnetization curve. The short magnetic lifetime is due to the strong interaction with the substrate electrons, as already observed for Ho and Er atoms on metal surfaces. ${ }^{11}$ To investigate whether the degree of hybridization between graphene and the supporting metallic substrate plays a role in the magnetization stability of the Dy atoms, we have investigated the magnetic properties of single Dy atoms on graphene/Ru(0001) (Figure S8b). The XAS line shape reveals the marked presence of two characteristic peaks at $1289.8 \mathrm{eV}$ and $1291.8 \mathrm{eV}$, indicating the presence of more than one atomic species with different $4 f$ occupation. Similar to the Dy atoms on $\operatorname{Ir}(111)$, this system exhibits paramagnetic behavior and short spin lifetime, deduced from the absence of hysteresis in the magnetization curve acquired at $1289.8 \mathrm{eV}$ and shown in Figure 4b. The magnetization curve acquired at $1291.8 \mathrm{eV}$ also shows paramagneic behavior.

\section{Bibliography}

(1) Hupalo, M.; Liu, X.; Wang, C.-Z.; Lu, W.-C.; Yao, Y.-X.; Ho, K.-M.; Tringides, M. C. Adv. Mater. 2011, 23, 2082.

(2) Liu, X.; Wang, C.-Z.; Hupalo, M.; Lin, H.-Q.; Ho, K.-M.; Tringides, M. C. Crystals 2013, 3, 79-111.

(3) Song, C.-L.; Sun, B.; Wang, Y.-L.; Jiang, Y.-P.; Wang, L.; He, K.; Chen, X.; Zhang, P.; Ma, X.-C.; Xue, Q.-K. Phys. Rev. Lett. 2012, 108, 156803.

(4) N’Diaye, A. T.; Coraux, J.; Plasa, T. N.; Busse, C.; Michely, T. New J. Phys. 2008, 10, 043033.

(5) Pichler, S.; Bodnarchuk, M. I.; Kovalenko, M. V.; Yarema, M.; Springholz, G.; Talapin, D. V.; Heiss, W. ACS Nano 2011, 5, 1703. 
(6) Thole, B. T.; van der Laan, G.; Fuggle, J. C.; Sawatzky, G. A.; Karnatak, R. C.; Esteva, J.-M. Phys. Rev. B 1985, 32, 5107-5118.

(7) Gatteschi, D.; Sessoli, R. Angew. Chem. Int. Ed. 2003, 42, 268-297.

(8) Uldry, A.; Vernay, F.; Delley, B. Phys. Rev. B 2012, 85, 125133.

(9) Chilton, N. F.; Collison, D.; Mclnnes, E. J. L.; Winpenny, R. E. P.; Soncini, A. Nat. Commun. 2013, 4, 2551.

(10) Baldovi, J. J.; Clemente-Juan, J. M.; Coronado, E.; Gaita-Ariño, A. Inorg. Chem. 2014, 53, 11323.

(11) Donati, F.; Singha, A.; Stepanow, S.; Wäckerlin, C.; Dreiser, J.; Gambardella, P.; Rusponi, S.; Brune, H. Phys. Rev. Lett. 2014, 113, 237201. 